\title{
An X-ray spectral survey of BL Lac objects
}

\author{
P. Barr ${ }^{1}$, P. Giommi ${ }^{1,2}$, A. Pollock ${ }^{1}$, G.Tagliaferri ${ }^{1}$, D. Maccagni ${ }^{3}$ and B. Garilli ${ }^{3}$ \\ ${ }^{1}$ EXOSAT Observatory, $\quad 2$ On leave from $\quad{ }^{3}$ IFC-CNR, via Bassini 15, \\ Astrophysics Division, ESA IFC-CNR, via Bassini 15, 20133 Milan, Italy \\ ESTEC, Postbus 299, 20133 Milan, Italy \\ 2200AG Noordwijk, \\ The Netherlands
}

\section{Introduction}

A wide variety of X-ray spectral forms has been reported in BL Lac objects. Concave spectra, i.e. a steep soft X-ray spectrum with a flat high energy tail, have been reported in a few of the brightest BL Lacs (e.g Urry 1986). Conversely, convex spectra (steep hard X-rays, flat soft X-ray spectrum) have also been reported, sometimes in the same objects (Madejski 1985 , Barr et al 1988, George et al 1988). The high energy tails have usually been invoked as a signature of synchrotron-self-Compton emission. Two conflicting interpretations of the convex spectra have been made. Urry et al (1986) suggest absorption by a partially ionised medium, probably intrinsic to the BL Lac object, following the identification of an Oxygen absorption trough in the Einstein OGS spectrum of PKS 2155-304 by Canizares and Kruper (1984). Conversely, Barr et al (1988) attribute the hard X-ray steepening to energy loss mechanisms operating on a synchrotron source.

The largest sample of moderate resolution, broad-band X-ray spectra of BL Lac objects compiled to date is in the EXOSAT archive. We have re-analysed all the EXOSAT data of BL Lac objects in a systematic manner and we summarise here our preliminary results.

\section{Observations and data analysis}

During its three-year lifetime EXOSAT observed 35 BL Lac objects, many of them more then once. Of these, seventeen were detected, and not confused, in the EXOSAT medium energy array (ME), allowing measurement of their X-ray spectra. A total of 66 observations of these seventeen sources yielded useful X-ray spectra (see table 1).

Various spectral models were folded through the LE/ME detector response and fitted to the data for each observation independently. We have summed the $\chi^{2}$ for all observations for each continuum model in order to estimate the statistical significance of any deviation from a single power law for this sample. The resulting $\chi^{2}$ and probability of exceeding that $\chi^{2}$ are given in table 2. In order to eliminate any possible systematic effects in the data extraction and spectral fitting, a control sample of EXOSAT observations of eight Seyferts and broad line radio galaxies were analysed in an identical manner. 
Table 1

\begin{tabular}{|l|c|c|l|c|c|}
\hline Source & $\begin{array}{c}\text { No. of } \\
\text { obs. }\end{array}$ & $\begin{array}{c}2-6 \mathrm{keV} \\
\text { countrate \# }\end{array}$ & Source & $\begin{array}{c}\text { No. of } \\
\text { obs. }\end{array}$ & $\begin{array}{c}2-6 \mathrm{keV} \\
\text { countrate }\end{array}$ \\
\hline $3 \mathrm{C} 66 \mathrm{~A}$ & 2 & $0.27-0.34$ & $1 \mathrm{E} 1402.3+0416$ & 2 & $0.13-0.2$ \\
$1 \mathrm{E} 0317.0+1835$ & 2 & $0.48-0.67$ & $1 \mathrm{E} 1415.6+2557$ & 1 & 0.44 \\
$1 \mathrm{H} 0323+023$ & 2 & $0.6-0.8$ & $1 \mathrm{H} 1427+42^{*}$ & 2 & $2.15-3.4$ \\
1H0414+009 & 4 & $0.9-1.8$ & Mkn 501 & 10 & $0.5-3.2$ \\
PKS 0521-365* & 2 & 0.67 & I Zw 186* & 2 & $0.57-0.62$ \\
PKS 0548-32* & 3 & $1.4-2.1$ & 3C 371 & 2 & $0.15-0.3$ \\
PKS 0754+100 & 1 & 0.37 & PKS 2005-489* & 5 & $0.14-4.9$ \\
Mkn 421* & 14 & $0.44-9.9$ & PKS 2155-304* & 9 & $1.45-15.9$ \\
Mkn 180 & 3 & $0.12-0.82$ & & & \\
\hline
\end{tabular}

\# 1 count s $\mathrm{s}^{-1} \sim 10^{-11} \mathrm{ergs} \mathrm{cm}^{-2} \mathrm{~s}^{-1} \quad$ *see text

Table 2

\begin{tabular}{|c|c|c|c|c|c|}
\hline Model & $\chi^{2} /$ d.o.f. & probability & Model & $\chi^{2} /$ d.o.f. & probability \\
\hline Power law & $2344 / 1834$ & $4.5 \times 10^{-15}$ & $\begin{array}{c}\text { power law, } \\
\text { hard tail }\end{array}$ & $2274.5 / 1690$ & $5.0 \times 10^{-20}$ \\
\hline $\begin{array}{c}\text { cutoff } \\
\text { power law }\end{array}$ & $1898 / 1762$ & 0.012 & $\begin{array}{c}\text { broken } \\
\text { power law }\end{array}$ & $1717.3 / 1690$ & 0.32 \\
$\begin{array}{c}\text { power law, } \\
\text { O edge }\end{array}$ & $1855.7 / 1690$ & $2.8 \times 10^{-3}$ & $\begin{array}{c}\text { power law, } \\
\text { O line }\end{array}$ & $1962.4 / 1690$ & $4.0 \times 10^{-6}$ \\
\hline
\end{tabular}

\section{Conclusion}

A single power law cannot reproduce the EXOSAT spectra of these BL Lac objects; rather, a continuum which steepens at high energies is required (this effect is not present in the control sample of Seyferts). In addition, the spectral steepening seen in these EXOSAT data cannot be due to an Oxygen absorption feature superimposed on a single power law, of the kind seen by Canizares \& Kruper in PKS 2155-305 and postulated by Urry et al for several more BL Lac objects; such a model is excluded at the $99.7 \%$ confidence level and is $1 / 100$ th as probable as the broken power law (an Oxygen feature superimposed on a broken power law cannot be excluded but is not required; the spectral steepening seen must be intrinsic to the continuum). In seven of these objects individually - those marked by an asterisk in table 1 - application of the F-test indicates that the broken power law is preferred over a single power law at the $\geq 99 \%$ confidence and in several cases is required in individual observations. No evidence for hard tails was found, either in the sample as a whole or in individual objects. Finally, it should be noted that a broken power law is not necessarily the only valid model and other curved continuum models may also be relevant.

Barr, P., Giommi, P. \& Maccagni, D., 1988, Ap.J., 324 , L11

Canizares,C.R. \& Kruper, J., 1984, Ap.J., 278 , L99

George, I.M., Warwick, R.S. \& Bromage, G.E., 1988, M.N.R.A.S., 232 ,793

Madejski, G., 1985, Ph.D. Thesis, Harvard University

Urry, M., 1986, in The physics of accretion onto compact objects, Springer-Verlag, p. 357

Urry, M., Mushotzky, R.F. \& Holt, S.S., 1986, Ap.J., 305 , 369 


\section{DISCUSSION}

WORRALL Do you observe any $X$-ray spectral differences between radioselected and $X$-ray-selected BL Lac objects?

BARR The $X$-ray selected $B L$ Lacs tend to be brighter in the hard $X$-rays, but our sample contains both X-ray (11 objects) and radio-selected (6) BL Lacs. Of the seven objects which show a spectral break individually, five are $X$-ray selected and two radio-selected (the other radio-selected objects are fainter than these seven $\mathrm{BL}$ Lacs). Because our hard X-ray sample is effectively flux-limited, it will be difficult to search for systematic differences between the two sub-classes.

ELVIS Since you observed many of the same objects as HEAO1-A2 in much the same energy range, do you have any suggestions as to why the EXOSAT ME data give such different results?

BARR The HEAO-1 A2 and EXOSAT ME detectors do cover somewhat different energy ranges in that, for weak sources like these, $A 2$ was sensitive out to energies above $230 \mathrm{keV}$ while EXOSAT only goes to $\sim 10 \mathrm{keV}$. Thus, if high energy tails only emerged above $10 \mathrm{keV}, \mathrm{HEAO}-1$ would have seen them, but we would not. (With the exception of Mkn 421 and PKS 2155-304, which were quite bright - we would have seen the high energy tails reported by HEAO- 1 if they had been present during our observations: we did not.) As for HEAO-1 not detecting the high energy turnover, no one ever tested those models against the HEAO-1 data!

Another point to bear in mind is source confusion - both the $A 2$ and ME detectors are passively collimated. Confusion is not really a problem with EXOSAT because we have simultaneous imaging with the LE (for instance, I didn't use the 2A $1219+305$ data because I know its confused in the ME - and was also confused with HEAO-1 and the Einstein MPC). Confusion can be a problem with HEAO-1, especially in view of the fact that the LED had a wider field of view than the MED! 